\section{APRENDIZAJE Y DESARROLLO EN LA PRIMERA INFANCIA}

\section{EARLY CHILDHOOD LEARNING AND DEVELOPMENT}

\author{
María Teresa Moreno Zavaleta \\ mariamorenoz@unife.edu.pe
}

\begin{abstract}
RESUMEN
Durante la educación temprana, concentramos nuestros esfuerzos en enseñar al niño lo que tiene que aprender y no nos fijamos en lo que el niño es capaz de aprender por sí mismo, los niños aprenden a través de la interacción con los adultos, con sus pares, con los objetos, a través del juego y los proyectos de aprendizaje. Desde que nace un niño está en la capacidad de aprender y desarrollarse, los adultos cuidadores y la maestra de inicial juegan un papel crucial, conociendo al niño en acción para ofrecerle un entorno seguro, enriquecido y pertinente. Este artículo presenta una reflexión de los conceptos de cuidado, así como el de educación que está vinculado a los conceptos de aprendizaje y desarrollo, transitando por diferentes marcos teóricos. También da a conocer cómo la maestra puede utilizar herramientas sencillas en clase para promover aprendizajes a través de proyectos realizados por los niños. En la medida que los adultos cuidadores -incluida la maestra de inicial- identifican las necesidades e intereses de los niños y conocen cómo aprende el niño, según las diversas teorías del conocimiento y del desarrollo infantil, podrán contribuir a mejorar los aprendizajes en su contexto escolar o en su entorno familiar y comunitario.
\end{abstract}

PALABRAS CLAVE

Aprendizaje, desarrollo, cuidado, educación infantil, interacciones de calidad.

\section{ABSTRACT}

During early education, we concentrate our efforts to teach the child what he has to learn, and we do not look at what the child can learn for himself, children learn through interaction with adults, with their peers, with objects, to through the game, and learning projects. From the birth of a child, he can learn and develop. Adult caregivers and the preschooler teacher play a crucial role, knowing the child in action to offer a safe, enriched, and suitable environment. This article presents an analysis of the concepts of care/education and theories of learning and development through different theoretical frameworks. It also makes them know how the teacher can use simple tools in class to promote children's education through projects. To the extent that adult caregivers, including the teacher, identify the needs and interests of children and know how the child learns according to different theories of knowledge and child development, they can contribute to improving children's learning in the context school or family and community environment.

KEYWORDS

Learning, development, care, early childhood education, quality interactions.
A compañar a un niño desde su nacimiento implica comprender qué significa: cuidar y educar; es decir, dos acciones que deben ser proporcionadas por los responsables de su cuidado y educación (menciono en plural porque el cuidado del niño no es exclusivo de una sola persona). El cuidado humano, puede ser descrito como una forma instintiva de proteger y cuidar la vida, como lo hace un mamífero con su cría; pero existen, además, otras condiciones y características que marcan las diferencias con las demás especies, entre ellas, está la capacidad del cuidador para entender que el cuidado no se trata solo de proteger a su cría del entorno, sino que la condición humana del recién nacido hace que sea reconocido como un individuo único, con posibilidades de pensar, sentir, actuar y trascender en su entorno a medida que crece y se desarrolla.

Bajo esta primera premisa, surge también otra variable que consiste en la concepción que tienen los cuidadores sobre quién es el niño, un sujeto solo con necesidades o un sujeto con derechos que requiere crecer y desarrollarse en un entorno seguro y amigable, rodeado de otros seres humanos. El concepto de infancia ha evolucionado a través de la historia, pero es a partir de la firma de la Convención de los Derechos del Niño que existe una forma socialmente aceptada para mirar al niño como un ser humano que necesita de un adulto que le ayude a aprender y desarrollarse como tal. 
Ahora bien, si es en la relación con los otros seres humanos cómo se construye el nuevo ser, entonces la interacción se convierte en un principio fundamental desde el inicio de la vida; por tanto, es imprescindible fijar nuestra atención en la forma cómo interactuamos, en qué momento lo hacemos, si logramos una interacción de calidad y si esta interacción es recíproca y armoniosa.

Los momentos del cuidado, constituyen un espacio privilegiado para las interacciones, son estos momentos que el adulto puede aproximase al niño e iniciar un diálogo de miradas, gestos y sonidos. Según sea la sensibilidad del adulto, mayor oportunidad tendrá el niño para expresar lo que siente, lo que vive y lo que explora. Los cuidados de calidad contribuyen a dar al niño seguridad para que descubra sus propios pensamientos y sentimientos, experimentando diversas experiencias para emprender nuevos desafíos y utilizar lo aprendido como base para sus iniciativas y proyectos de acción.

El cuidado, por tanto, es una forma de educar, por lo que es imposible cuidar sin educar o educar sin cuidar, ambas son prácticas sociales inseparables y necesarias para el desarrollo pleno del ser humano. En la Meta 4.2 del Objetivo de Desarrollo Sostenible (ODS) el numeral 4, referido a la educación, establece que los Estados se comprometieron a asegurar que todas las niñas y todos los niños tengan acceso a la atención y al desarrollo en la primera infancia y educación preescolar de calidad, a fin de que estén preparados para la enseñanza primaria. Los conceptos clave que deben ser medidos para lograr el indicador de la meta incluyen la calidad del cuidado y la educación, el acceso a programas, y el desarrollo y aprendizaje del niño al comienzo de la vida escolar (UNESCO, 2019).

En ese sentido, es necesario conceptualizar el cuidado y la educación, desde el punto de vista de la teoría existente relacionada con cómo aprenden los niños y cómo se desarrollan. Ricardo Baquero (2017), menciona que "el desarrollo y el aprendizaje deben ser comprendidos como procesos de apropiación mutua o recíproca de los sujetos y las prácticas culturales de las que ellos participan".

La calidad del cuidado, por tanto, va a definir la forma como el niño aprende sobre sí mismo y sobre el sujeto que lo cuida, lo que lo llevará progresivamente a poner en práctica su autocuidado e inclusive a desarrollar habilidades para cuidar a otros. Podríamos decir que mientras cuidamos al niño, este va desplegando sus habilidades, se va desarrollando y al mismo tiempo va aprendiendo en la interacción con el otro y los objetos físicos de su entorno.

Ahora bien, ¿cómo aprende un niño en sus primeros años de vida? ¿el aprendizaje es interno o externo al sujeto que aprende? ¿es necesario que los niños alcancen un buen desarrollo para aprender o el aprendizaje permite su desarrollo? ¿cuál es el rol de la maestra de educación inicial para promover el aprendizaje y desarrollo en la primera infancia? ¿qué debe conocer dicha maestra sobre su entorno familiar y comunitario? Estas son algunas preguntas a las que pretendo dar respuesta en el presente artículo.

\section{¿Cómo aprende un niño en sus primeros años?}

La evidencia científica nos devela que, si un niño recibe en sus primeros años de vida, cuidados y educación de calidad, podrá sentar las bases de su desarrollo humano (Oates, 2007). Por ello, cuando un niño se enfrenta con un problema utilizará, de manera estratégica, los recursos internos y externos desarrollados desde su nacimiento, los que le permitirán actuar de manera coherente, reflexiva, sistemática y propositiva, en pocas palabras, convertirse en un ser competente (Joannert, Barrete, Masciotra \& Yaya, 2008).

Por tal motivo, para que el aprendizaje se dé es necesario preparar las condiciones necesarias, contar con espacios seguros y materiales pertinentes según sea la edad del niño. 
El niño también aprende a través del juego, cuando se interesa por algo y disfruta lo que hace jugando con las personas o los objetos que tiene a su alrededor. Otra forma como los niños nos muestran que aprenden es a través de los proyectos de aprendizaje que se realizan con la participación de ellos mismos, en los cuales son el centro del aprendizaje y protagonistas de su propio desarrollo.

\section{¿El aprendizaje es interno o externo al sujeto que aprende?}

Para responder esta pregunta, debemos analizar cómo es un niño desde que nace, cómo llega al mundo. Según las neurociencias, el bebé llega provisto de una impronta por descubrir y aprender, viene dotado de una estructura física, mental y emocional que le permite dar a conocer lo que necesita, lo que le interesa y esto varía en cada niño según sus características individuales. Sin embargo, si las condiciones son adversas, las oportunidades disminuyen y tiene menos posibilidades de desarrollar plenamente su potencialidad, por tanto, el entorno es crucial para el desarrollo infantil; es decir, para expandir sus capacidades de explorar, comprender e interactuar con sus pares, sus cuidadores y los objetos que lo rodean (Shonkof \& Philipps, 2000).

Desde la biología podemos observar, que el ser humano nace equipado para generar mecanismos para atraer al cuidador; por ejemplo, a través de su olor, el cual al ser especial enternece al igual que su piel suave, su mirada tierna, etc., todo esto es justo lo que necesita para que el cuidador sea sensible y se "enamore del bebé", se vincule, se acerque a él y lo proteja.

Pero lo que marcará la diferencia con otros seres vivos, es el uso del pensamiento, es decir la capacidad que tiene el ser humano de pensar, lo que será posible gracias a las múltiples experiencias que se le proporciona de manera dosificada y armónica, protegiendo su estado emocional. Este proceso es el sustento para su pensamiento simbólico, crítico y creativo.
Las zonas superiores del cerebro humano en los primeros meses de vida preparan al bebé para pensar y razonar, dotándolo así de ser el único ser vivo con estas capacidades. Asimismo, a lo largo de las primeras semanas de vida, se van creando en el cerebro humano nuevas rutas neuronales que permitirán que las distintas capacidades del pequeño se vayan desarrollando.

Debido a que el proceso es progresivo, porque se ajusta a la estructura y madurez que tiene cada niño de acuerdo con su edad y a su ritmo, es necesario, conocer cómo se da el proceso de desarrollo evolutivo del niño para asegurar las condiciones pertinentes que contribuyan a su desarrollo y al mismo tiempo a su aprendizaje.

Ahora bien, no es suficiente generar, crear o preparar las condiciones para que el niño pueda desarrollarse, sino que, al mismo tiempo, debemos asegurarnos cómo el niño interactúa con su entorno y cómo acompañamos este proceso de adaptación y apropiación tanto del entorno físico como del entorno social.

Para entender cómo se da este proceso, es necesario comprender como piensa el niño, qué habilidades cognitivas pone en juego, cómo utiliza los procesos psicológicos elementales y superiores. Es decir, en este momento, estaríamos describiendo cómo aprende el niño, observando la forma cómo utiliza las habilidades $\mathrm{y}$ conocimientos que ha adquirido para resolver un problema.

Este proceso afirma lo que nos dice Vigotsky (citado por Baquero) "el aprendizaje despierta una serie de procesos evolutivos internos capaces de operar sólo cuando el niño está en interacción con las personas de su entorno y en cooperación con algún semejante. Una vez que se han interiorizado, estos procesos se convierten en parte de los logros evolutivos independientes del niño" (Vigotsky, 1998, pp. 138-139).

Veamos un ejemplo: Nicolás es un niño de dos años que busca estrategias para alcanzar un objeto que está en un lugar lejano a él, dado 
que su intención es movida por la curiosidad de jugar con dicho objeto, que le parece atractivo, empieza a utilizar su seguridad postural para mover un banco de madera, utiliza su equilibrio postural para subir al banco y sus destrezas motoras para coger con sus manos el objetivo y usarlo como él desea.

En este ejemplo, Nicolás hace uso de sus habilidades motoras, las cuales son necesarias para moverse, coger un banco y subirse en el, así como para alcanzar el objeto deseado. Hasta allí nos ha demostrado que puede conseguir lo que desea, utilizando sus habilidades motoras, pero su interés o motivación por conocer al objeto hace que ponga a prueba sus habilidades para conseguirlo utilizando los medios que tiene a su alcance.

Ahora bien, para que Nicolás se sienta seguro de emprender una tarea posiblemente riesgosa para algunos, porque podría caer del banco, es necesario considerar cómo fue construyendo su seguridad y autonomía, dos competencias importantes que le permitieron conseguir su objetivo. Pero, a su vez, está demostrando que es capaz de planificar una acción y dominar esquemas de conocimiento de su entorno; es decir, demuestra que tiene inteligencia sensorio motriz. En este caso, la significación no viene dada por la presencia directa del objeto, sino por las acciones a las que se asimila lo que se observa (Castorina, 2019).

Por otro lado, no podemos dejar de analizar que Nicolás no se encuentra solo, para que pueda desarrollar sus habilidades y capacidades ha sido necesaria la presencia de un adulto, que lo acompaña, que interactúa con él, que le prepara el espacio para que pueda moverse con libertad y que está atento a sus necesidades e intereses.

\section{¿Es necesario que los niños alcancen un buen desarrollo para aprender o el aprendizaje permite su desarrollo?}

La literatura nos menciona que el desarrollo de un niño es integral, no se puede estudiar de manera fragmentada. Asimismo, todo lo que hace un niño tiene relación con su dimensión emocional, cognitiva y física, por ejemplo, para que haya desarrollado el manejo de sus emociones, primero ha tenido que conocer qué son las emociones, expresarlas y tener un adulto que le permita regular dichas emociones. La presencia del adulto es fundamental tanto para lograr un buen desarrollo como para lograr un buen aprendizaje.

El aprendizaje y desarrollo son conceptos íntimamente vinculados, es decir, uno depende del otro y viceversa, no tendríamos que esperar que el niño esté desarrollado para que pueda aprender, ni que aprenda para que pueda desarrollarse, ambas situaciones se pueden dar por separado.

Cuando decimos que el desarrollo precede al aprendizaje, usualmente los maestros atribuyen este concepto a Piaget, sin embargo, es necesario comprender que puede confundirse con el término maduración. Es decir, cuando el niño está maduro puede aprender mejor, esta maduración biológica está más relacionada a procesos de desarrollo neurológico que al desarrollo motor. Los ciclos madurativos en cierta medida preceden a los ciclos de aprendizaje.

Mientras que, cuando decimos que el aprendizaje precede al desarrollo, estamos pensando en Vigotsky quien afirmaba que cuando el aprendizaje se da estimula y hace avanzar el desarrollo. Sin embargo, tanto los procesos de desarrollo como de aprendizaje se dan de manera interactiva, se complementan entre sí y deben ser estudiados de manera conjunta (Vasco Uribe \& Henao López, 2008).

\section{¿Cómo los docentes de educación inicial pueden contribuir al aprendizaje y desarrollo de los niños?}

Primero, es necesario regresar a los conceptos de cuidado y educación; es decir, las maestras de inicial deben conocer que el cuidado es básico para el desarrollo y aprendizaje que se requiere 
en la educación del niño. Para ello, la maestra tiene que involucrarse en el cuidado del niño y trabajar de manera coordinada con la familia, para que las prácticas de crianza del hogar le aseguren un buen desarrollo.

Desde un enfoque médico, es posible que enfoquemos nuestro interés en lograr que el niño tenga una buena salud y nutrición, pero eso no es suficiente, un niño necesita estar bien físicamente pero también estar alerta, sentirse seguro en su entorno para moverse en libertad y explorar sin miedo lo que tiene a su alcance.

Si la maestra no conoce o no comprende el contexto donde vive el niño, es posible que no pueda identificar las necesidades e intereses de los niños. Partir de las necesidades del niño y de sus intereses permite que éste sea protagonista de su propio aprendizaje y desarrollo.

También es imprescindible que la maestra comprenda cómo aprende y cómo se desarrolla el niño, incluso desde que está en el vientre materno, esto le permitirá distinguir los estadios de su desarrollo, respetar los ritmos evolutivos propios de su edad y le permitirá proporcionar al niño elementos necesarios para enriquecer su entorno ya sea con gestos, palabras o con objetos sin llegar a sobre estimularlo o sobrecargarlo. He aquí lo complejo de la educación de un niño que implica darle a cada uno lo que requiere según su edad, retarlo sin cansarlo u obligarlo a hacer algo que no puede hacerlo porque aún no se encuentra maduro para realizarlo.

Cuando comprendemos los procesos del desarrollo motor, por ejemplo, vemos que para un niño aprender a caminar es un proceso largo, -generalmente un niño requiere en promedio entre 10 a 15 meses para dar sus primeros pasos de manera autónoma-. Asimismo, requiere entre 24 a 36 meses para comunicar verbalmente lo que quiere y requiere entre 36 a 72 meses para gestionar sus emociones.

Los procesos de desarrollo no son lineales y la forma como adquiera sus niveles de desarrollo va a depender de quién acompañe al niño y de su contexto. Asimismo, es muy importante comprender cómo el niño interactúa, qué mecanismos pone en marcha, cómo es capaz de aprender dentro y fuera de escuela (Rogoff B., 2012), guiado por la cultura y los valores sociales (Rogoff B., 1997).

Cada niño, por tanto, va a desarrollarse de manera diferente, pero todos los niños logran desarrollarse y aprender, a algunos les resultará más sencillo y otros requerirán más esfuerzos, pero todos llegarán a hacerlo en la medida de que cuenten con un adulto sensible que acompañe este proceso y un entorno cultural accesible y con quien pueda interactuar libremente.

\section{De la teoría a la práctica}

La mayoría de las maestras hemos aprendido mucho sobre las teorías de aprendizaje y sobre las teorías del desarrollo infantil; sin embargo, estas teorías solo se pueden comprender cuando las analizamos al observar a un niño en acción.

Por tanto, es necesario analizar las teorías que ya han sido estudiadas y que han evolucionado a través de los años. Propongo detenernos a analizar la teoría del filósofo inglés John Locke, quien en el siglo XVII rechazaba la noción de innatismo y argumentaba que todas las ideas nos llegan a través de la experiencia sensorial, las ideas pasaban de simples a complejas, esta teoría revolucionaria para su época, constituyó, según Brunner, los cimientos de los planteamientos modernos en el tema de la construcción del conocimiento (Daros, 2000).

Permitir que un niño se aproxime a su cuidador y a los objetos a través de la exploración sensorial es fundamental para iniciar cualquier proceso de aprendizaje. En la medida que el niño va conociendo a las personas y objetos que lo rodean según sea su contexto o cultura, va adquiriendo conocimientos que utiliza, relacionándolos entre sí, todo esto da origen a su capacidad de razonar, al desarrollo de su pensamiento crítico y pese a que aún no puede expresar con exactitud qué 
es lo que está pensando, empieza a cuestionarse sobre el funcionamiento de las cosas y sobre las actitudes de las personas, empieza a conocer las leyes de la materia, observa que si tira un objeto, este no volverá a sus manos de manera natural, al menos que una persona se lo vuelva a dar o al menos que el objeto rebote como es el caso de la pelota.

\section{¿El niño piensa como científico?}

Otra concepción importante que debe conocer la maestra de inicial es que los niños, a medida que se desarrollan, piensan y cuando piensan, lo hacen utilizando sus conocimientos $\mathrm{y}$ experiencias, así como su curiosidad intuitiva por aprender, la misma curiosidad que motiva a los científicos para investigar. Esta habilidad permite al niño generar hipótesis, experimentar y sacar sus propias conclusiones, aunque no necesariamente utilizando el método científico.

Cuando el niño está muy pequeño, posiblemente experimente de manera más sencilla y concreta, pero conforme crece y explora el mundo, se va haciendo nuevas preguntas y va actuando a través de la experimentación para comprobar sus hipótesis y tener sus propias conclusiones.

Veamos un ejemplo para comprender mejor este tema.

\section{La transformación de la calabaza ${ }^{1}$}

La maestra de inicial desea desarrollar con los niños de 4 años la competencia: "Indaga mediante métodos científicos para construir conocimientos" (competencia que se encuentra en el Currículo de Educación Básica Regular del Perú).

Para desarrollar esta tarea, la maestra coloca una calabaza en un lugar visible del aula con la intención de despertar curiosidad e interés por el fruto. Cuando un grupo de niños la vieron, hicieron una serie de cuestionamientos: ¿Quién ha dejado esta cosa aquí? ¿Qué es esta cosa tan extraña? ¿Por qué la han dejado aquí? Algunos pensaron que podría ser una pelota, pero no lo era, porque al cargarla se dieron cuenta que era muy pesada y las pelotas rebotan.

Cuando examinaron la calabaza, se dieron cuenta que había una grieta y al acercarse para observar que tenía dentro se percataron que había pepas. Es una fruta gigante dijo uno de los niños, entonces la maestra que estaba observándolos preguntó: ¿Por qué creen que es una fruta gigante? Uno de los niños dijo: porque me parece haberla visto en el mercado, y otros dijeron: sí, es una fruta grande y la venden en el mercado.

La maestra siguió preguntando: ¿saben cómo se llama?, y los niños se quedaron en silencio, uno dijo zapallo y otro dijo no, no es un zapallo.

¿Entonces alguien dijo, maestra diga ¿cómo se llama esta fruta y cómo llegó aquí? La maestra dijo esta fruta se llama calabaza y nos ha venido a visitar para que aprendamos de ella.

¿Qué podemos aprender de esta calabaza?

La vamos a abrir para comerla dijo Ana, pero antes de abrirla hay que dibujarla dijo Juan, y cada niño expresó algo para hacer con la calabaza.

Antes de abrirla, la maestra les dijo que podrían observarla algunos días, mientras tanto podemos hacer un dibujo colectivo.

No era la primera vez que los niños de 4 años realizaban el ejercicio de observar un objeto y representarlo, lo habían hecho en proyectos anteriores. Pero, esta vez, la maestra les propuso hacer 
un dibujo colectivo, por ello les pidió compartir primero sus observaciones y ponerse de acuerdo para ver qué características incluirían.

En este caso, la maestra está atenta a las observaciones y preguntas de los niños, les hace otras preguntas, escucha las respuestas y provoca una situación problemática para que los niños se interesen por aprender de la calabaza.

¿Qué tipos de aprendizaje se pueden dar a partir de esta situación? ¿Qué otras acciones deben promover para lograr movilizar capacidades que conlleven a desarrollar la competencia?

\section{Reflexión sobre la problemática y las posibles propuestas de solución}

La problemática plantea la utilización del método científico que surge ante la inquietud de los niños por averiguar de qué se trata el elemento con el que se encontraron. Se logra evidenciar cómo ponen en juego sus conocimientos previos y a partir de ellos intentan averiguar con qué se encontraron.

La maestra, a partir de las preguntas realizadas por los niños, podría elaborar hipótesis junto a los niños en cuanto al sabor de la calabaza, sus usos, el crecimiento, etc., y comprobarlas buscando información en diferentes soportes.

Luego se realizaría una prueba de hipótesis; es decir, comprobar si la hipótesis propuesta es válida o no, esto se puede comprobar experimentado el sabor, midiendo la calabaza, comparando, etc.

Aquellas hipótesis que no se pudieran comprobar, como el caso de "la calabaza es una pelota" y alguien dijo que "no era posible porque el objeto pesaba y las pelotas rebotan", los mismos niños con sus explicaciones podrían rebatir las hipótesis propuestas.

La metodología de aprendizaje propuesta por la maestra es un aprendizaje por descubrimiento, apropiado para los niños de inicial. Lo que Brunner llama "Modelo cognitivo de aprendizaje de conceptos por la vía de la identificación o definición de atributo" (Camargo Uribe \& Hederich Martínez, 2010). Esta estrategia de "no instrucción directa" les permite a los chicos construir conocimiento conjuntamente.

En esta problemática se aprecia el rol del docente, como un mediador del aprendizaje, al traer la calabaza provoca el interés de los niños, pero al mismo tiempo escucha las preguntas que se hacen los niños y, a la vez, genera preguntas para que los niños puedan pensar y sacar sus propias conclusiones.

Lo más interesante es habilitar la pregunta, que los niños tengan ante quién preguntarse (ese es un rol fundamental de un maestro) y suspender la respuesta para permitirles que investiguen (Schujman, 2019). Lo importante es sostener este tipo de actividades en el tiempo, que pasen a ser una suerte de rutina de pensamiento. Al partir de las hipótesis de los niños, se está trabajando desde el método hipotético deductivo (Bruner, 1991).

El aprendizaje que señala la problemática puede enriquecerse con lo que se señala en el enfoque Reggio-Emilia, quien invita a los docentes a salirnos de nuestro rol tradicional, permitiéndonos ver quiénes podemos llegar a ser si es que estamos dispuestos a asumir los riesgos: cambiar nuestra imagen acerca de los niños es un requisito fundamental.

Este enfoque parte del concepto que los niños no son recipientes vacíos a los que hay que llenar con conocimientos, sino seres con un gran potencial, competentes y capaces de construir sus propias teorías. 
Loris Malaguzzi, fundador de esta filosofía, ha descripto claramente los derechos de los niños: ser reconocidos como sujetos individuales y, a la vez, partícipes de una sociedad, construir sus propias experiencias, siendo participantes activos en la organización de sus identidades, habilidades y autonomía, a través de la relación con sus propios pares, con los adultos, con las ideas, con los objetos y con los eventos, tanto reales como imaginarios. Además, Malaguzzi plantea un pensamiento circular: sentir, pensar, actuar, estas tres acciones se realizan conjuntamente (Hoyuelos \& Cabanellas, 1996).

El rol del docente es acompañar a los niños, descubriendo sus intereses y sus ideas, respondiendo a sus necesidades, respetando sus tiempos y no ajustarlos a los institucionales, dándoles lugar a sus preguntas, creando una atmósfera comunicativa y afectiva en la sala para que puedan expresarse en libertad, promover la reflexión y atender a la diversidad.

Un aspecto importante para este proceso de aprendizaje es la documentación. Por un lado, constituyen la "memoria" de lo ocurrido: a partir de ellas los docentes podemos reflexionar acerca de nuestras prácticas, monitorear el proceso de aprendizaje de nuestros alumnos, interpretar sus pensamientos y ayudarnos a decidir la dirección que debe tomar una secuencia pedagógica. Es decir, nos permite seguir aprendiendo $\mathrm{y}$ capacitándonos como profesionales, ya que es un ejercicio que requiere de una fina observación y análisis. Además, nos abre las puertas a nuevos cuestionamientos, a la búsqueda de soluciones y a compartir nuestras experiencias con el resto de los docentes, creando espacios de reflexión conjunta, que sin duda nos hacen aprender, perfeccionarnos y sobre todo, crecer como educadores y como personas.

Por otro lado, se transforman en material educativo para otros docentes, pero no como la simple descripción de una actividad, sino con el valor agregado de sus resultados durante la práctica, sus logros y sus aciertos, permitiéndonos mejorarla $\mathrm{y}$, a la vez, avanzar en nuestra comprensión sobre el pensamiento de los niños.

Además, la documentación sostiene la memoria de los niños, ofreciéndoles la oportunidad de revisar su propio proceso, de encontrar la confirmación o la negación y de poder auto corregirse; les permite compararse con otros y escuchar comparaciones de otros, invita a auto evaluaciones y evaluaciones grupales, genera conflictos cognitivos y discusiones (Carretero, 1985). A su vez, la documentación provee una excelente oportunidad para padres, les permite conocer no solo lo que su hijo hace, sino también cómo y por qué, a revisar no simplemente el producto, sino también el proceso.

Para realizar una buena documentación es necesario conocer a quienes van dirigidas las documentaciones (docentes, niños, padres, futuros docentes) y también conocer el proceso para documentar (observar, registrar, interpretar los procesos, evaluar).

Otro aspecto importante para incluir en la problemática es la incorporación de nuevos conocimientos a través de la investigación: insertar el conocimiento en el entorno inmediato del niño, desde el espacio familiar, el barrio, la plaza, la escuela, entre otros.

\section{¿Qué debe conocer la maestra sobre su entorno familiar y comunitario?}

Termino este artículo reflexionando sobre lo que la maestra de inicial requiere conocer del entorno familiar y comunitario del niño. La maestra es un agente educativo, pero no conseguirá aprendizajes si cree que lo que hace es suficiente, se necesita trabajar de la mano con la familia y la comunidad. Para ello, es necesario conversar con todos los miembros de la familia para identificar qué prácticas de crianza cotidianas se realizan en el hogar y la comunidad, promueven y/o limitan el desarrollo del niño, qué condiciones externas se pueden mejorar y cómo contribuir de manera conjunta a lograr que los niños aprendan y se desarrollen de manera óptima. 
Conocer el entorno familiar es una responsabilidad del educador de niños durante la primera infancia, conocer qué piensan, cómo fueron criados, qué prácticas de cuidado y de educación se dan en el hogar, escucharlos más y dejar de darles recetas, son formas de promover un trabajo en conjunto.

Si se promueve la reflexión con cada familia y se generan espacios de reflexión con la comunidad, es posible conocer más al niño y apreciar cómo con la participación de todos se pueden resolver problemas comunes, intercambiar experiencias y contribuir a darle al niño prácticas adecuadas y coherentes según sea su edad y partiendo de la visión del niño que queremos para nuestra sociedad.

La familia y la comunidad cumplen su rol educador, pueden contribuir junto con la escuela a lograr que niños y niñas desarrollen al máximo todo su potencial.

\section{REFERENCIAS}

Bruner, J. (1991). Actos de significado. Madrid: Alianza Editorial.

Camargo Uribe, A. \& Hederich Martínez, C. (2010). Jerome Bruner: Dos teorías, dos formas de significar, dos enfoques para la

\section{CONCLUSIONES}

El aprendizaje y el desarrollo en la primera infancia requieren de un adulto consciente para modificar, intervenir o limitar dichos procesos. La calidad de las interacciones del niño con los adultos, con sus pares y con los objetos de su entorno, son los que determinarán su progreso y su actuar en la vida cotidiana. La familia, la escuela y la comunidad son espacios que contribuyen al aprendizaje y desarrollo en la medida de que cuenten con una visión compartida sobre la formación del niño que queremos para la sociedad en que vivimos.

En la medida que los adultos cuidadores, incluida la maestra, identifiquen las necesidades e intereses de los niños y conozcan cómo aprenden, podrán contribuir a mejorar los aprendizajes adquiridos en su contexto escolar o en su entorno familiar y comunitario. enseñanza de la ciencia. Psicogente, 13(24), 329-346. Recuperado de: https://www. redalyc.org/pdf/4975/497552357008.pdf

Carretero, M. (1985). Aprendizaje y desarrollo cognitivo. En J. Mayor Sanchez. Actividad 
humana y procesos cognitivos (pp. 145160). Madrid: Alhambra.

Castorina, J. A. (26 de diciembre de 2019). Diploma Superior en Constructivismo y Educación, cohorte 26. Recuperado de: FLACSO VIRTUAL: https:// virtual.flacso.org.ar/mod/book/view. php?id $=662611 \&$ chapterid $=885361$

Daros, W. R. (2000). La construcción de los conocimientos en los niños según el empirismo de John Locke. Invenio, 3(4-5), 57-59. Recuperado de: https://www.redalyc. org/pdf/877/87730503.pdf

Hoyuelos, A. \& Cabanellas, I. (1996). Malaguzi y el valor de lo cotidiano. Congreso de Pamplona Diciembre-96, (pp. 1-11). Pamplona. Recuperado de: http://www. waece.org/biblioteca/pdfs/d091.pdf

Joannert, P., Barrete, J., Masciotra, D. \& Yaya, M. (15 de diciembre de 2008). La competencia como organizadora de los programas de formación: hacia un desempeño competente. Recuperado de: https://recyt.fecyt.es/index. php/profesorado/index https://www.ugr. es/ recfpro/rev123ART3.pdf

Oates, J. (2007). Relaciones de apego, la calidad del cuidado en los primeros años. Reino Unido: The open University. Recuperado de: https://issuu.com/bernardvanleerfoundation/ docs/relaciones_de_apego_la_calidad_del_ cuidado_en_los_primeros_anos

Rogoff, B. (1997). Los tres planos de la actividad sociocultural: apropiación participativa, participación guiada y aprendizaje. En J.
Wertsh, P. Del Río \& A. Álvarez. La mente sociocultural. Aproximaciones teóricas aplicadas. (pp. 111-128). Madrid: Fundación Infancia y Aprendizaje.

Rogoff, B. (2012). Aprender sin lecciones: oportunidades para expandir el conocimiento. Infancia y Aprendizaje, 35(2), 233-252.

Schujman, G. (abril de 2019). Recuperado de: https://www.flacso.org.ar/el-desafio-desuspender-la-respuesta-para-habilitar-lapregunta/

Shonkof, J. P. \& Philipps, D. A. (2000). De las Neuronas al Vecindario: la Ciencia del Desarrollo Infantil Temprano. Washington DC: Prensa de la Academia Nacional.

UNESCO. (2019). SDG 4 Data Digest, How to Produce and Use the Global and Thematic Education Indicators. Montreal, Quebec, Canadá: UNESCO Institute for Statistics. Recuperado de: http:/uis.unesco.org/sites/ default/files/documents/sdg4-data-digest2019-en_0.pdf

Vasco Uribe, C. E. \& Henao López, G. C. (2008). Elementos y modelos del desarrollo: Una revisión del concepto. En J. LarreamendyJoerns, R. Puche-Navarro \& A. Restrepo Ibiza. Claves para pensar el cambio: Ensayos sobre psicología del desarrollo. (p. 240). Bogotá: Ediciones Uniandes. Recuperado de: http://observatorio.ascofapsi.org.co/static/ documents/Claves_para_pensar_el_cambio.pdf

Vigotsky, L. (1988). El desarrollo de los procesos psicológicos superiores. Madrid: Crítica Grijalbo. 